\title{
ISOMETRISABLE GROUP ACTIONS
}

\author{
ITAÏ BEN YAACOV AND JULIEN MELLERAY
}

(Communicated by Kevin Whyte)

\begin{abstract}
Given a separable metrisable space $X$, and a group $G$ of homeomorphisms of $X$, we introduce a topological property of the action $G \curvearrowright X$ which is equivalent to the existence of a $G$-invariant compatible metric on $X$. This extends a result of Marjanović obtained under the additional assumption that $X$ is locally compact.
\end{abstract}

\section{INTRODUCTION}

This paper grew out of the following question: given a metrisable topological space $X$, and a homeomorphism $g$ of $X$, how can one determine whether there exists a distance inducing the topology of $X$ and for which $g$ is an isometry? More generally, it is interesting to determine when there exists a compatible invariant distance for an action by homeomorphisms of some group $G$ on $X$. When this happens we say that the action $G \curvearrowright X$ is isometrisable.

When $X$ is compact, this problem is well understood, and various characterisations are available - for instance, in that case an action $G \curvearrowright X$ is isometrisable if and only if it is equicontinuous, in the sense that for any open $U \subseteq X \times X$ containing the diagonal $\Delta_{X}$, there exists an open $V \subseteq X \times X$ containing $\Delta_{X}$ and such that for all $g \in G$ one has $(g \times g) V \subseteq U$. One way to prove this is to note that, if the latter property holds, then the sets $G \cdot V$ form a countably generated uniformity which is compatible with the topology and admits a basis of invariant entourages, and such a uniformity comes from a $G$-invariant metric (as a general reference about uniformities and the basic facts about them used in this paper, the reader may consult Chapter 8 in [Eng89]). One could equivalently formalise the previous condition by saying that $G$ generates a relatively compact subgroup of the group of homeomorphisms of $X$, endowed with the topology of uniform convergence for some compatible distance on $X$ (here the nontrivial direction follows from the Arzelà-Ascoli theorem, or by considering averages of any compatible metric against the Haar measure of $G$ ).

Beyond that, only the locally compact case seems to be addressed in the literature. Marjanović Mar69 appears to be the first with a significant result in this direction. In order to formulate it, we recall that, if $\mathcal{F}$ is a family of continuous maps from a topological space $X$ to a topological space $Y, \mathcal{F}$ is said to be evenly continuous if for all $x \in X$, all $y \in Y$ and all open $V \ni y$, there exists an open

Received by the editors June 30, 2014 and, in revised form, October 28, 2015.

2010 Mathematics Subject Classification. Primary 22F05, 37B05.

Key words and phrases. Group action, isometric group action, topological space, metric space.

This research was supported by ANR project GruPoLoCo (ANR-11-JS01-008). 
$U \ni x$ and an open $W$ with $y \in W \subseteq V$ and such that

$$
\forall f \in \mathcal{F} f(x) \in W \Rightarrow f(U) \subseteq V .
$$

Theorem (Marjanović Mar69). Let $X$ be a locally compact separable metrisable space, and $f$ be a homeomorphism of $X$. Then there is a compatible distance for which $f$ is an isometry if, and only if, the family $\left\{f^{n}: n \in \mathbf{Z}\right\}$ is evenly continuous from $X$ to its Alexandrov compactification.

This result was slightly extended by Borges Bor71 and Kiang Kia73; it follows from Kiang's work that Marjanovic's result extends to arbitrary groups acting on locally compact separable metrisable spaces (though that fact is not explicitly formulated in Kia73] and could also be deduced from Marjanović's argument, it is a direct consequence of the main theorem of [Kia73]). One obstacle to extend these results beyond the locally compact case is the presence of the Alexandrov compactification in the statement; another is that Marjanović's and Kiang's arguments rely heavily on compactness. To address the first issue, one might try considering a stronger property than even continuity.

Definition (Royden Roy88). The action $G \curvearrowright X$ is topologically equicontinuous if, for any $x, y \in X$ and any open subset $V \ni y$, there exists open subsets $W \ni x$ and $y \in U \subseteq V$ such that

$$
\forall g \in G \quad(g W \cap U \neq \emptyset) \Rightarrow g W \subseteq V .
$$

It is obvious that, if $G \curvearrowright X$ is isometrisable, then it is topologically equicontinuous. It is also not hard to check that, when $X$ is locally compact, even continuity of $G$ as a family of maps from $X$ to its Alexandrov compactification is equivalent to topological equicontinuity of $G$ as a family of maps from $X$ to itself. Topological equicontinuity is a strong assumption, and we discuss some consequences in the second section. It appears not to be sufficient for isometrisability of the action $G \curvearrowright X$, leading us to consider an even stronger property.

Definition. We say that $G \curvearrowright X$ is uniformly topologically equicontinuous if, for any $y \in X$ and any open $V \ni y$, there exists an open $U$ with $y \in U \subseteq V$ and such that for all $x \in X$ there exists an open neighbourhood $W$ of $x$ satisfying

$$
\forall g \in G(g W \cap U \neq \emptyset) \Rightarrow g W \subseteq V .
$$

Our main result is the following.

Theorem. Let $X$ be a second countable metrisable space, and $G$ be a group acting on $X$ by homeomorphisms. Then the action $G \curvearrowright X$ is isometrisable if, and only if, it is uniformly topologically equicontinuous.

Rather than directly defining a $G$-invariant compatible metric under the assumption of uniform topological equicontinuity, our argument proceeds by building a countably generated uniformity with a basis of $G$-invariant entourages, then using the fact that such a uniformity is generated by $G$-invariant pseudometrics, and finally using second countability to subsume this family into one $G$-invariant metric.

\section{TOPOLOGICAL EQUICONTINUITY}

Throughout the text $X$ stands for a separable metrisable space, and $G$ is a group of homeomorphisms of $X$. 
Lemma 1.1. Assume that $G \curvearrowright X$ is topologically equicontinuous. Assume that $x_{n} \rightarrow x$ and $y_{n} \rightarrow y$ in $X$, and let $\left(g_{n}\right) \in G^{\mathbf{N}}$ be such that $g_{n} x_{n} \rightarrow y$. Then $g_{n}^{-1} y_{n} \rightarrow x$.

In particular, $g_{n} x \rightarrow y$ if and only if $g_{n}^{-1} y \rightarrow x$; thus a topologically equicontinuous action is minimal if and only if it is topologically transitive.

Proof. Fix $U$ open containing $x$, and find $V$ open contained in $U$ and containing $x, W$ open containing $y$ such that $(g W \cap V \neq \emptyset) \Rightarrow g W \subseteq U$ for any $g \in G$. For $n$ large enough $x_{n} \in V$ and $y_{n}, g_{n} x_{n} \in W$, so $g_{n}^{-1} W \subseteq \bar{U}$, and in particular $g_{n}^{-1} y_{n} \in U$, as desired.

To see why topological transitivity implies minimality, assume the action is topologically transitive (that is, for any nonempty open $U, V$ there exists $g \in G$ such that $g U \cap V \neq \emptyset$ ) and pick $x, y \in X$. By assumption, there exist $g_{n} \in G$ and $x_{n} \in X$ such that $x_{n}$ converges to $x$ and $g_{n} x_{n}$ converges to $y$. Hence $g_{n}^{-1} y$ converges to $x$, showing that the orbit of $y$ is dense.

Proposition 1.2. Assume that $G \curvearrowright X$ is minimal. Then $G \curvearrowright X$ is isometrisable if, and only if, it is topologically equicontinuous.

Proof. One implication is clear. For the other, assume that $G \curvearrowright X$ is topologically equicontinuous, and denote by $\tau$ the topology of $X$. Consider the family of sets of the form $G \cdot U^{2} \subseteq X^{2}$ where $U$ varies over all nonempty open sets in $X$.

Since the action is minimal, $G \cdot U^{2}$ contains the diagonal. Given such a set $G \cdot U^{2}$, find an open $\emptyset \neq V \subseteq U$ such that $g V \cap V \neq \emptyset \Rightarrow g V \subseteq U$. Assume now that $(x, y),(y, z) \in G \cdot V^{2}$, say $x, y \in h_{1} V$ and $y, z \in h_{2} V$. Then $h_{1}^{-1} y \in V \cap h_{1}^{-1} h_{2} V$, hence $h_{1}^{-1} h_{2} V \subseteq U$, so $h_{1}^{-1} z \in U$. Thus both $h_{1}^{-1} x$ and $h_{1}^{-1} z$ belong to $U$, and $(x, z) \in G \cdot U^{2}$.

Thus the sets $G \cdot U^{2}$ form a basis of entourages for a uniformity, which is metrisable by a $G$-invariant distance $d$ since it is countably generated by $G$-invariant entourages, and we claim that it is compatible with the topology on $X$. Since each $G \cdot U^{2}$ is open, $d$ is continuous. Conversely, assume that $x_{n} \rightarrow^{d} x$. For every neighbourhood $U$ of $x$ we have $\left(x_{n}, x\right) \in G \cdot U^{2}$ for all $n$ large enough, giving rise to a sequence $\left(g_{n}\right)$ such that $g_{n} x_{n} \rightarrow x$ and $g_{n} x \rightarrow x$. By Lemma 1.1 we have $x_{n} \rightarrow x$. Therefore $d$ is a compatible metric.

When the action $G \curvearrowright X$ is assumed to be transitive, the above result appears as an exercise in Royden Roy88.

Next we introduce the "topological ergodic decomposition" associated to $G \curvearrowright X$.

Definition 1.3. For $x, y \in X$, let $[x]=\overline{G x}$ and say that $x \sim y$ if $x \in[y]$.

Lemma 1.4. Assume that $G \curvearrowright X$ is topologically equicontinuous. Then the relation $\sim$ is a closed equivalence relation on $X$ (i.e., it is an equivalence relation which is closed as a subset of $X \times X)$.

In particular, $[x]=\{y: x \sim y\}$ and $x \sim y$ if and only if $[x] \cap[y] \neq \emptyset$.

Proof. It is clear that $\sim$ is transitive and reflexive, and when $G \curvearrowright X$ is topologically equicontinuous it is also symmetric, by Lemma 1.1. In order to see that it is closed, assume that $\left(x_{n}, y_{n}\right) \rightarrow(x, y)$ in $X^{2}$, where $x_{n} \sim y_{n}$ for all $n$. If $U \ni x$ is open, for $n$ large enough we have $x_{n} \in U$, and since $x_{n} \sim y_{n}$, there exist $g_{n} \in G$ such that $g_{n} y_{n} \in U$. We thus construct a sequence $\left(g_{n}\right)$ with $g_{n} y_{n} \rightarrow x$. By Lemma 1.1 we have $g_{n}^{-1} x \rightarrow y$ and $y \sim x$, as desired. 
Consequently the quotient space which we denote by $X / / G$ is Hausdorff. If the action $G \curvearrowright X$ is isometrisable, then this quotient must be metrisable.

Lemma 1.5. Assume that $G \curvearrowright X$ is topologically equicontinuous. Then the projection map $\pi: X \rightarrow X / / G$ is open.

Proof. Let $U \subseteq X$ be open, $x \in U$ and $y \sim x$. Then $G y \cap U \neq \emptyset$, or equivalently, $y \in G U$. It follows that the open set $G U$ is the $\sim$-saturation of $U$, so $\pi U$ is open.

\section{UNIFORM TOPOLOGICAL EQUICONTINUITY AND ISOMETRISABILITY}

Metrisability of $X / / G$ is obviously a necessary condition for the action $G \curvearrowright X$ to be isometrisable. Outside the realm of locally compact spaces, this seems to require a stronger hypothesis than mere topological equicontinuity.

Definition 2.1. We say that $G \curvearrowright X$ is uniformly topologically equicontinuous if for any $x \in X$ and any open $V \ni x$ there exists an open $U$ with $x \in U \subseteq V$ such that for all $y \in X$ there exists an open $W_{y} \ni y$ satisfying

$$
\forall g \in G\left(g W_{y} \cap U \neq \emptyset\right) \Rightarrow g W_{y} \subseteq V .
$$

When the conditions above are satisfied, we say that $U$ witnesses uniform topological equicontinuity for $x, V$.

This definition is obtained by inverting two quantifiers in the definition of topological equicontinuity, and is still a necessary condition for isometrisability of $G \curvearrowright$ $X$.

Proposition 2.2. Assume that $G \curvearrowright X$ is uniformly topologically equicontinuous. Then $X / / G$ is metrisable.

Proof. Since $X$ is second countable so is $X / / G$, and it will suffice to prove that $X / / G$ is regular. In other words, we need to prove that given a closed $G$-invariant $F \subseteq X$ and $x \notin F$, there exist open sets $U \ni x$ and $W \supseteq F$ such that $U \cap G W=\emptyset$. We choose $U$ which witnesses uniform topological equicontinuity for $x, X \backslash F$, and for each $y \in F$ we let $W_{y} \ni y$ be the corresponding neighbourhood. If there existed $y \in F$ and $g \in G$ such that $g W_{y} \cap U \neq \emptyset$, then $g W_{y} \subseteq X \backslash F$ and in particular $g y \notin F$, a contradiction. Therefore $U \cap \bigcup_{y \in F} G W_{y}=\emptyset$, which is enough.

Given Marjanović's result recalled in the introduction, the following fact is worth mentioning. (If one merely wishes to prove that $X / / G$ is metrisable when $X$ is locally compact and the action is topologically equicontinuous, a much shorter argument exists.)

Proposition 2.3. Let $X$ be a locally compact separable metrisable space, and $G$ a group acting on $X$ by homeomorphisms. The following conditions are equivalent:

(1) $G \curvearrowright X$ is uniformly topologically equicontinuous.

(2) $G \curvearrowright X$ is topologically equicontinuous.

(3) $G$, seen as a family of maps from $X$ to its Alexandrov compactification $X^{*}$, is evenly continuous.

Proof. Note that (3) is equivalent to saying that, for all $x \in X$ and $y \in X^{*}$, if $\left(x_{i}\right)$ converges to $x$ and $\left(g_{i} x\right)$ converges to $y$, then $\left(g_{i} x_{i}\right)$ also converges to $y$.

The implication (11) $\Rightarrow$ (2) is by definition. To see that (2) implies (3), assume that there exists $x \in X$ and a compact $K \subseteq X$ such that for all open $U \ni x$ and 
for all compact $L \supseteq K$ there is $g \in G$ such that $g(x) \notin L$ and $g(U) \cap K \neq \emptyset$. From this we may build a sequence $\left(x_{i}\right)$ converging to $x$ and elements $g_{i} \in G$ such that $g_{i}(x) \rightarrow \infty$ and $g_{i}\left(x_{i}\right) \rightarrow k \in K$. This is incompatible with (2).

It remains to prove that (3) $\Rightarrow$ (1). We again proceed by contradiction and assume that $G \curvearrowright X$ is not uniformly topologically equicontinuous but $G$ is an evenly continuous family of maps from $X$ to $X^{*}$. By assumption, there exists $y \in X$ and an open $V \ni y$ such that for any open $U$ with $y \in U \subseteq V$ there exists $x \in X$ such that for all open $W \ni x$ there exists $g \in G$ with both $g W \cap U \neq \emptyset$ and $g W \nsubseteq V$. Letting $U$ vary over a basis of open neighbourhoods of $y$, we obtain a sequence $\left(x_{i}\right)$ witnessing the above condition; up to extractions we see that there are two cases to consider:

- $\left(x_{i}\right)$ converges to some $x \in X$. Then there exists sequences $\left(g_{i}\right)$ and $\left(y_{i}\right),\left(z_{i}\right)$ converging to $x$ such that $g_{i} y_{i}$ converges to $y$ and $g_{i} z_{i}$ lives outside $V$. Up to some extraction, we may assume that $g_{i} x$ and $g_{i} z_{i}$ both converge in $X^{*}$, and the fact that $g_{i} y_{i}$ and $g_{i} z_{i}$ have different limits shows that even continuity must be violated at $x$.

- $\left(x_{i}\right)$ converges to $\infty$, and for all compact $K$ there exists $I$ such that for all $i \geq I$ and all $g$ one has $g x_{i} \notin K$ (otherwise, replacing $x_{i}$ by some $g_{i} x_{i}$ and going to a subsequence we would be in the situation of the first case above). Letting $U$ be a relatively compact neighbourhood of $y$, we see that for $i$ large enough we have $\overline{G x_{i}} \cap \bar{U}=\emptyset$. Then the even continuity of $G$ implies that there must exist some neighbourhood $W$ of $x_{i}$ such that $G W \cap \bar{U}=\emptyset$ (by essentially the same argument as above), which contradicts the choice of $x_{i}$.

Definition 2.4. Let $\mathcal{U}$ be an open cover of $X$. We say that it is $G$-invariant if for any $U$ one has $U \in \mathcal{U} \Rightarrow g U \in \mathcal{U}$.

A $G$-basis of a $G$-invariant open cover $\mathcal{U}$ is a subset $\mathcal{B}$ such that all elements of $\mathcal{U}$ are of the form $g B$ for some $B \in \mathcal{B}$.

We say that a $G$-invariant open cover $\mathcal{U}$ is $G$-locally finite if it admits a $G$-basis $\mathcal{B}$ such that for any $x \in X$ there exists a neighbourhood $A$ of $x$ (not necessarily belonging to $\mathcal{U})$ such that $\{B \in \mathcal{B}: \exists g \in G g B \cap A \neq \emptyset\}$ is finite.

Lemma 2.5. Assume that $G \curvearrowright X$ is uniformly topologically equicontinuous. Then any $G$-invariant open cover admits a $G$-locally finite open refinement.

Proof. Let $\mathcal{U}$ be a $G$-invariant open cover. Let also $\pi: X \rightarrow X / / G$ denote the open quotient map. Since $X / / G$ is metrisable, it is paracompact (see e.g. Theorem 4.4.1 in Eng89), so we can find a locally finite refinement $\mathcal{V}$ of $\pi \mathcal{U}$. For any $V \in \mathcal{V}$, pick some $U_{V} \in \mathcal{U}$ such that $\pi\left(U_{V}\right) \supseteq V$, and set $W_{V}=U_{V} \cap \pi^{-1}(V)$. Let $\mathcal{W}$ be the $G$-invariant open cover with $G$-basis $\left\{W_{V}: V \in \mathcal{V}\right\}$. By construction $\mathcal{W}$ is an open cover and refines $\mathcal{U}$.

Now pick any $x \in X$. There is an open neighbourhood $O$ of $\pi(x)$ which meets only finitely many elements of $\mathcal{V}$. If $W_{V}$ is such that $g W_{V} \cap \pi^{-1}(O) \neq \emptyset$ for some $g \in G$, then $V \cap O \neq \emptyset$, so $\mathcal{W}$ is $G$-locally finite.

Notation 2.6. To an open cover $\mathcal{U}$ of $X$ we associate an entourage $\mathcal{E}(\mathcal{U})=\bigcup_{U \in \mathcal{U}} U^{2} \subseteq$ $X^{2}$.

Lemma 2.7. Assume that $G \curvearrowright X$ is uniformly topologically equicontinuous. Let $\mathcal{U}$ be a $G$-invariant open cover of $X$. Then there exists a $G$-invariant open refinement 
$\mathcal{V}$ of $\mathcal{U}$ with the property that for all $x, y, z \in X$, if $(x, y),(y, z) \in \mathcal{E}(\mathcal{V})$, then $(x, z) \in \mathcal{E}(\mathcal{U})$.

Proof. Uniform topological equicontinuity of the action enables us to find a $G$ invariant open refinement $\mathcal{W}$ of $\mathcal{U}$ with the property that for all $W \in \mathcal{W}$ there exists $U(W) \in \mathcal{U}$ containing $W$ such that for all $y \in X$ there exists an open $C_{W, y} \ni y$ satisfying $g C_{W, y} \cap W \neq \emptyset \Rightarrow g C_{W, y} \subseteq U(W)$. Using Lemma 2.5 we may assume that $\mathcal{W}$ is $G$-locally finite and $\mathcal{B}$ is a $G$-basis of $\mathcal{W}$ witnessing that property. We let $\mathcal{V}$ consist of all open sets $V$ such that for all $g \in G$ and $B \in \mathcal{B}$ :

$$
g V \cap B \neq \emptyset \Rightarrow g V \subseteq U(B) .
$$

Given $x \in X$ there exists an open $A \ni x$ such that $\mathcal{B}_{A}=\{B \in \mathcal{B}: g B \cap A \neq \emptyset\}$ is finite, so $x \in A \cap \bigcap_{B \in \mathcal{B}_{A}} C_{B, x} \in \mathcal{V}$. Thus $\mathcal{V}$ is a cover, and it is clearly $G$-invariant and refines $\mathcal{U}$.

Assume now that $(x, y),(y, z) \in \mathcal{E}(\mathcal{V})$, say $x, y \in V_{1}$ and $y, z \in V_{2}$ where $V_{i} \in \mathcal{V}$. There exist some $B \in \mathcal{B}$ and $g \in G$ such that $g y \in B$, so $g V_{1} \cup g V_{2} \subseteq U(B)$ and $x, z \in g^{-1} U(B) \in \mathcal{U}$.

Lemma 2.8. Assume that $G \curvearrowright X$ is uniformly topologically equicontinuous, and fix $x \in X$. For any $G$-invariant open cover $\mathcal{U}$ there exists a $G$-invariant open refinement of $\mathcal{U}$ with $G$-basis $\mathcal{B}$ and $B \in \mathcal{B}$ such that for any $A \in \mathcal{B}$ different from $B$ and any $g \in G$ one has $x \notin g A$.

Proof. Pick $U \in \mathcal{U}$ such that $x \in U$. Using uniform topological equicontinuity, choose an open neighbourhood $V$ of $x$ such that for any $y \in X \backslash G U$ there exists an open set $W_{y}$ satisfying $g W_{y} \cap V=\emptyset$ for all $g \in G$. Refining if necessary, we may assume that each $W_{y}$ is contained in some element of $\mathcal{U}$; then $\left\{W_{y}: y \in\right.$ $X \backslash G U\} \cup\{U\}$ form a $G$-basis for a $G$-invariant open refinement of $\mathcal{U}$ with the desired property.

Lemma 2.9. Assume that $G \curvearrowright X$ is uniformly topologically equicontinuous. Then for any $x \in X$ there exists a continuous $G$-invariant pseudometric $d_{x}$ such that $d_{x}\left(x_{i}, x\right)$ converges to 0 if and only if $\left(x_{i}\right)$ converges to $x$.

Proof. Fix $x \in X$. Using Lemmas 2.8 and 2.7. we can build a sequence of $G$ invariant coverings $\mathcal{U}_{n}$ of $X$ with $G$-basis $\mathcal{B}_{n}$ with the following properties:

- For each $n$ there exists a unique $B_{n} \in \mathcal{B}_{n}$ such that $x \in G B_{n}$, and $\left\{B_{n}\right\}_{n}$ forms a basis of neighbourhoods of $x$.

- For all $n$, if $(y, z),(z, t) \in \mathcal{E}_{n+1}$, then $(y, t) \in \mathcal{E}_{n}$, where $\mathcal{E}_{n}=\mathcal{E}\left(\mathcal{U}_{n}\right)$.

As in the proof of Proposition [1.2 the family of entourages $\mathcal{E}_{n}$ gives rise to a uniformity which is moreover metrisable by a $G$-invariant pseudometric $d_{x}$. Since all entourages are open, $d_{x}$ is continuous.

Assuming that $d_{x}\left(x_{i}, x\right) \rightarrow 0$, for all $n$ there must exist some $U_{n} \in \mathcal{U}_{n}$ such that $x_{i}, x \in U_{n}$ for all $i$ large enough, and $U_{n}$ must be of the form $g_{n} B_{n}$. It follows that there exists a sequence $h_{i}$ with $h_{i} x_{i} \rightarrow x$ and $h_{i} x \rightarrow x$, so $x_{i} \rightarrow x$ by Lemma 1.1 .

Theorem 2.10. Let $X$ be a second-countable metrisable space, and let $G$ act on $X$ by homeomorphisms. Then $G \curvearrowright X$ is isometrisable if and only if it is uniformly topologically equicontinuous. 
Proof. One direction is clear, so we prove the other. Applying Lemma 2.9, we obtain a family of continuous $G$-invariant pseudometrics $\left(d_{x}\right)_{x \in X}$. Since $d_{x}\left(x, x_{i}\right) \rightarrow 0$ if and only if $x_{i} \rightarrow x$, for any open subset $U$ of $X$ and any $x \in U$, there exists $\varepsilon>0$ such that $d_{x}(x, y)<\varepsilon \Rightarrow y \in U$. By the Lindelöff property, we obtain that for any open $U \subseteq X$ there exists a countable subset $A \subseteq U$ and a family $\left(\varepsilon_{a}\right)_{a \in A}$ such that

$$
U=\bigcup_{a \in A}\left\{x: d_{a}(x, a)<\varepsilon_{a}\right\} .
$$

Applying this to a countable basis for the topology of $X$, we obtain a countable family of $G$-invariant pseudometrics which generate the topology, and this countable family may be subsumed into a single $G$-invariant metric.

\section{Complete And incomplete metrics}

In this section we assume that $G \curvearrowright X$ is isometrisable, and $X$ admits a compatible complete metric. A natural question is then: must $G \curvearrowright X$ admit a complete invariant metric?

The following observation is immediate.

Proposition 3.1. Assume that $G \curvearrowright X$ is minimal. Then there exists a complete compatible $G$-invariant distance on $X$ if and only if all compatible $G$-invariant distances are complete.

Proof. Assume that a compatible complete $G$-invariant distance exists, fix $x \in X$, and let $d$ be another $G$-invariant compatible distance. Then a sequence $\left(g_{i} x\right)$ is $d$-Cauchy if and only if for any neighbourhood $V$ of $x$ there exists $N$ such that $g_{i}^{-1} g_{j} x \in V$ for all $i, j \geq N$. This property does not depend on $d$ but only on the topology of $X$, so any $d$-Cauchy sequence of the form $\left(g_{i} x\right)$ must converge.

Given any $d$-Cauchy sequence $\left(x_{i}\right)$, the minimality of $G \curvearrowright X$ enables us to find $g_{i}$ such that $d\left(g_{i} x, x_{i}\right)<2^{-i}$ for all $i$. Then $\left(g_{i} x\right)$ is also $d$-Cauchy, hence convergent, and so is $\left(x_{i}\right)$.

The fact above is well known in the particular case when $G$ is a Polish group acting by left translation on itself (and the proof is the same). All Polish groups admit left-invariant compatible metrics, but not all of them admit such metrics which are also complete (and, if one such metric is complete, all of them are). For instance, the group $S_{\infty}$ of all permutations of the integers, endowed with its usual Polish topology, does not admit a compatible left-invariant complete metric.

The following simple example was suggested by C. Rosendal.

Example 3.2. There exists a Polish space $X$ and a $\mathbf{Z}$-action on $X$ which is isometrisable but which admits no complete invariant distance.

Proof. Let $r$ be an irrational rotation of the unit circle $\mathbf{S}$, and let $X=\mathbf{S} \backslash\left\{r^{i}(1)\right.$ : $i \in \mathbf{Z}\}$. Then $X$ is a $G_{\delta}$ subset of $\mathbf{S}$, hence Polish, and the restriction of $r$ to $X$ generates an isometrisable $\mathbf{Z}$-action; the metric on $X$ induced from the usual metric on $\mathbf{S}$ is both invariant and not complete, so there cannot exist an invariant complete metric on $X$.

As it turns out, the minimal case contains essentially all the obstructions to the existence of a complete invariant metric. 
Theorem 3.3. Assume that $X$ is completely metrisable and the action $G \curvearrowright X$ is isometrisable. Then there exists a compatible complete $G$-invariant distance on $X$ if and only if there exists such a distance on the closure of each $G$-orbit.

Proof. The condition is clearly necessary. Now assume that there exists a compatible complete metric on the closure of each $G$-orbit, and let $d$ be a $G$-invariant metric on $X$. By Proposition 3.1 the restriction of $d$ to each $[x]=\overline{G x}$ is complete. Also, since the projection map $X \rightarrow X / / G$ is open and open maps with range in a metrisable space preserve complete metrisability (see for instance Exercise 5.5.8(d) p. 341 in Eng89), there exists a complete distance $\rho$ on $X / / G$. Consider the new metric $d^{\prime}$ defined by

$$
d^{\prime}(x, y)=d(x, y)+\rho([x],[y])
$$

Clearly $d^{\prime}$ is $G$-invariant and compatible with the topology of $X$. Assume now that $\left(x_{n}\right)$ is $d^{\prime}$-Cauchy. Since $\rho$ is complete, $\left[x_{n}\right]$ must converge to $[x]$ for some $x \in X$, i.e., there exists a sequence $\left(g_{n}\right)$ such that $g_{n} x_{n} \rightarrow x$. By invariance, $d\left(x_{n}, g_{n}^{-1} x\right) \rightarrow 0$, so $\left(g_{n}^{-1} x\right)$ is a $d$-Cauchy sequence in $[x]$ which must converge to some $y$. Therefore $x_{n} \rightarrow y$ as well, concluding the proof.

\section{REFERENCES}

[Bor71] Carlos R. Borges, How to recognize homeomorphisms and isometries, Pacific J. Math. 37 (1971), 625-633. MR0305328 (46 \#4458)

[Eng89] Ryszard Engelking, General topology, 2nd ed., Sigma Series in Pure Mathematics, vol. 6, Heldermann Verlag, Berlin, 1989. Translated from the Polish by the author. MR1039321 (91c:54001)

[Kia73] Mo Tak Kiang, On some semigroups of mappings, Nederl. Akad. Wetensch. Proc. Ser. A 76=Indag. Math. 35 (1973), 18-22. MR0317287 (47 \#5834)

[Mar69] M. M. Marjanović, On topological isometries, Nederl. Akad. Wetnesch. Proc. Ser. A 72=Indag. Math. 31 (1969), 184-189. MR0246252 (39 \#7556)

[Roy88] H. L. Royden, Real analysis, 3rd ed., Macmillan Publishing Company, New York, 1988. MR $1013117(90 g: 00004)$

Institut Camille Jordan, CNRS UMR 5208, Université Claude Bernard - Lyon 1, 43 Boulevard du 11 novembre 1918, 69622 Villeurbanne Cedex, France

Institut Camille Jordan, CNRS UMR 5208, Université Claude Bernard - Lyon 1, 43 Boulevard du 11 novembre 1918, 69622 Villeurbanne Cedex, France 